\title{
Giant cardiac hydatid cyst: case presentation with radiologic survey and review of literature
}

\author{
Kumar $\mathbf{R}^{1}$, Kumar $\mathrm{A}^{2}$, Agrahari $\mathrm{NS}^{3}$, Jaiswal $\mathbf{G}^{4}$, Kundu $\mathrm{J}^{5}$
}

${ }^{1}$ Ravinder Kumar, Assistant Professor, Department of Radiodiagnosis, Geetanjali Medical College and Hospital, Geetanjali University, India; ${ }^{2}$ Amit Kumar, second year DM (Cardiology) resident, Department of Cardiology, RNT Medical College and MB Hospital, RUHS University, India; ${ }^{3}$ Neha Singh Argahari; ${ }^{4}$ Gagan Jaiswal, first year MD residents, Department of Radiodiagnosis, Geetanjali Medical College and Hospital, Geetanjali University, India; ${ }^{5}$ yoti Kundu, Senior Lecturer, Department of Prosthodontics, Geetanjali Dental College and Hospital, Geetanjali University, India.

\begin{abstract}
Hydatid disease is mostly localised to liver and lungs. Cardiac involvement is a rare but potentially very serious complication of echinococcosis, constituting only $0.5-2 \%$ of all cases of hydatidosis. Cardiac hydatid cyst is a diagnostic and therapeutic challenge on account of highly variable clinical presentations and non-specific symptoms and often numerous unpredictable complications. We present a case report of 20-year-old man who was admitted to our hospital with chief complaint of palpitations and shortness of breath. Using baseline investigations like ECG, transthoracic echocardiography, ultrasonography, non-contrast computed tomography and magnetic resonance imaging, a giant multiloculated cystic lesion $(58.4 \mathrm{~mm} \times 43.3 \mathrm{~mm}$ ) was diagnosed in the apex of left ventricle. Serologic tests (hydatid cyst antibody) confirmed Echinococcus infection. Thoracic computed tomography with intravenous contrast was not performed because patient reported history of allergy to contrast. This case report is unusual as it is concerned with the description of a rare disease entity (large cardiac hydatid cyst). Its radiological appearances are discussed for early diagnosis and better understanding of the disease, together with a review of the literature.
\end{abstract}

Key words: computed tomography scan, echocardiography, electrocardiogram, hydatid cyst, magnetic resonance imaging, ultrasonography.

\section{INTRODUCTION}

$\mathrm{H}$ ydatidosis is a zoonotic parasitic disease caused by larval stage of Echinococcus granulosus, which is endemic in many sheep and cattle-raising countries. This disease is usually found in the liver (65\%) and lungs (25\%) but no organ of body is immune ${ }^{1-4}$. Location at unusual sites in the body can have atypical presentations and can pose a diagnostic challenge. A high index of suspicion, radiological investigations as well as histopathological examination is necessary in establishing the diagnosis of hydatid disease at unusual sites in the body. Cardiac involvement is a rare entity accounting for less than two percent of cases of echinococcosis ${ }^{3,5}$. Williams in 1836 first reported a case of cardiac hydatid disease ${ }^{6}$. The clinical presentation in cardiac hydatid cyst is usually

Address for correspondence

Dr. Ravinder Kumar

Assistant Professor

Department of Radiodiagnosis

Geetanjali Medical College and Hospital, Geetanjali University, Rajasthan, India.

E-mail: kundu19@yahoo.co.in insidious, but early diagnosis and management are very important due to risk of cyst perforation.

\section{CASE REPORT}

A 20-year-old man was admitted to our hospital with one month history of precordial pain, progressive dyspnoea, precipitated by minimal exertion and palpitations. The patient hailed from a rural community and had been in close contact with animals, never smoked, and had no other significant past medical history. His blood pressure was $80 / 60 \mathrm{~mm} \mathrm{Hg}$, his pulse rate was 115 beats/min and his heart sounds were normal. Other systemic physical examinations revealed nothing abnormal. Routine biochemical and haematological investigations were normal.

The chest X-ray (posterior-anterior) film showed an increased cardiothoracic index with a bulge on the left border of the cardiac silhouette. The left hilum was prominent with a round, well circumscribed mass seen within it. The electrocardiogram (ECG) exhibited T wave 
inversion resembling arrhythmia on left precordial leads and multiple ventricular premature complexes (VPCs). Holter monitoring showed run of nonsustained ventricular tachycardia (VT). Transthoracic echocardiography showed a huge multi-septate cystic mass within the apical region of left ventricle (Figure 1). His sonographic examination (Voluson E8, GE Healthcare) further suggested the presence of a giant cardiac hydatid cyst measuring $58.4 \mathrm{~mm} \times 43.3 \mathrm{~mm}$ in dimension (Figure 2). Parasitic serology of the patient was positive for anti-Echinococcus granulosus antibody by ELISA test. Intradermal Casoni's test and indirect haemagglutination tests were also positive. Diagnosis of cardiac hydatid cyst was confirmed and detailed structure was revealed by computed tomography scan (CT) and magnetic resonance imaging (MRI) findings. Since, patient was allergic to contrast, non-enhanced spiral computed tomography (NCCT) was done. NCCT thorax was performed on a 64-MDCT scanner (Somatom sensation 64, Siemens Healthcare). Figure 3 shows a well-defined large, rounded, multiseptate cystic lesion with hypodense content, partially calcified wall and multiple isodense septae within, noted in the apical part of left ventricle.

MRI was performed with 1.5-T system (Siemens Medical Healthcare) to know the exact anatomic location, morphology and nature of the internal and external structures relation of the cyst with cardiac chambers and confirming the extent of lesion. MRI depicted a characteristic rounded lesion appearing hypo intense on T1-weighted images (Figure 4a) and hyper intense centre with hypo intense periphery (pericyst) on T2-weighted images (Figure $4 \mathrm{~b}$ and c). Multiple intralesional daughter cysts were also seen on T2-weighted sequences.

The patient underwent cardiopulmonary bypass surgery and the large cardiac cyst was removed. Albendazole was started preoperatively and continued for six months after surgery. The patient recovered uneventfully, no evidence of cardiac abnormalities or recurrence was detected during the follow-up evaluation.

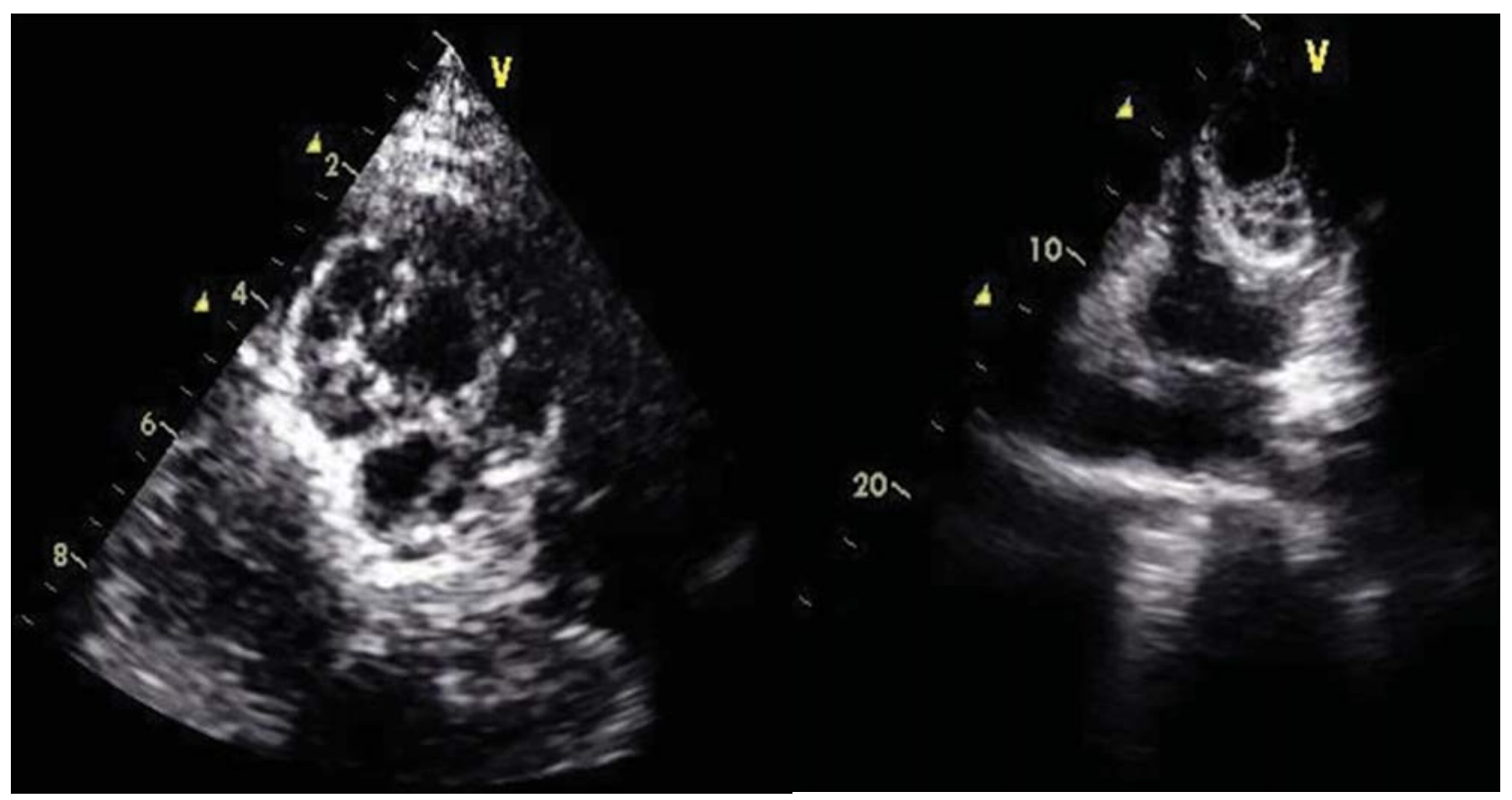

Figure 1: Transthoracic echocardiographic images showing large septate cystic mass in left ventricular apex. 


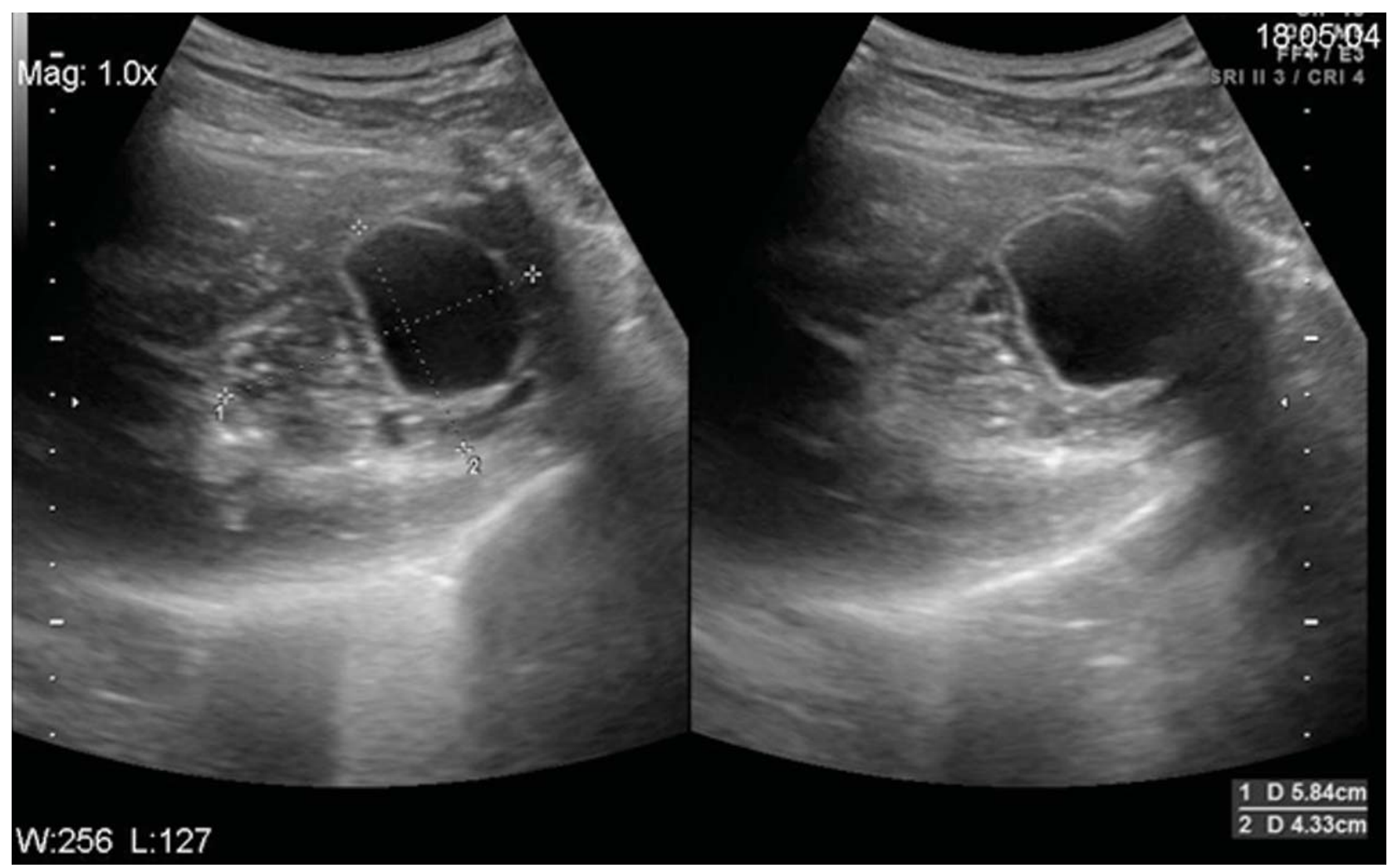

Figure 2: USG image showing a huge multi-septate cystic mass with dimension $58 \mathrm{~mm} \times 43 \mathrm{~mm}$ in the apical region of left ventricle of heart.

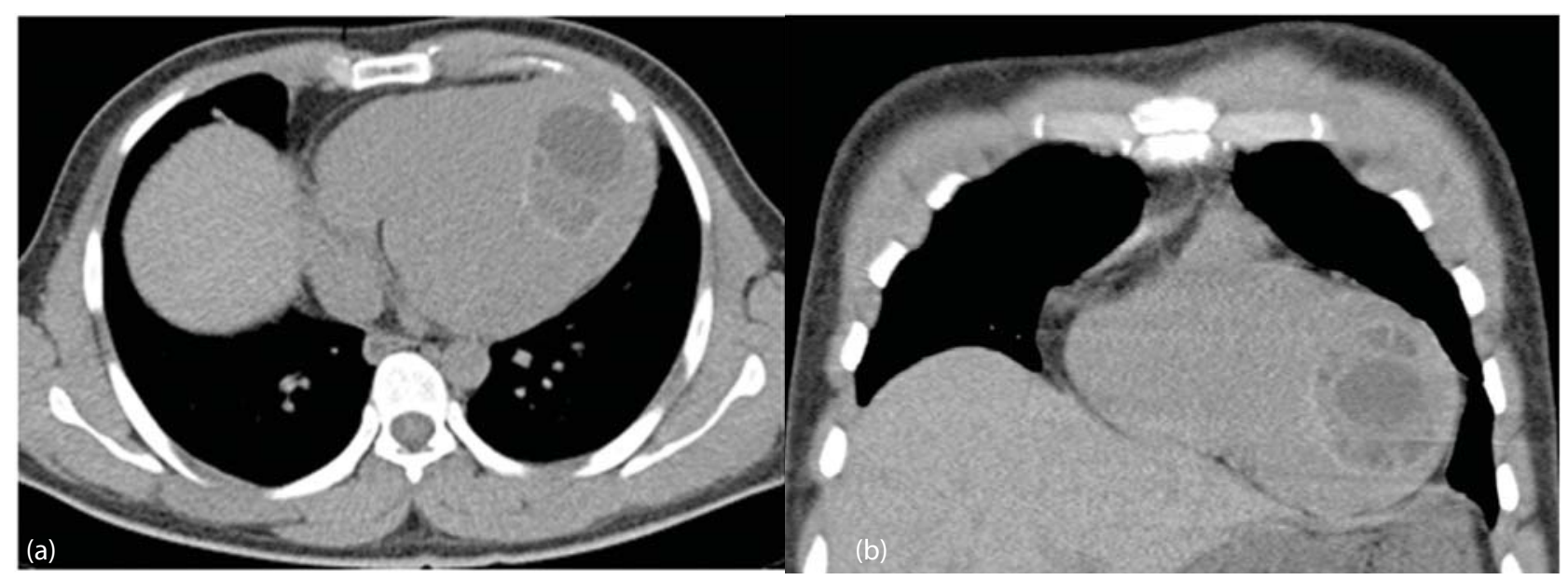

Figure 3: (a) Axial and (b) Coronal high resolution CT scan showing a well-defined large, rounded, multiseptate cystic lesion with hypodense content, partially calcified wall and multiple isodense septae within, noted in the apical part of left ventricle. 


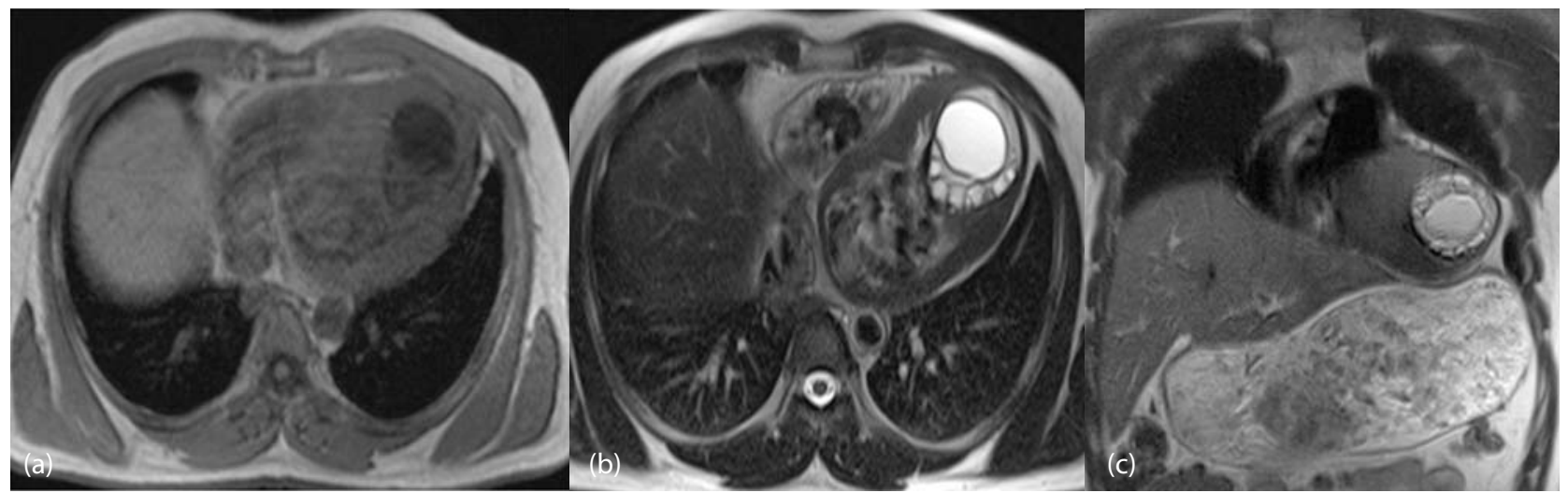

Figure 4: (a) Four-chamber dark-blood spin-echo T1-weighted MR image exhibiting large hypointense left ventricular hydatid cyst arising from apex. (b) and (c) T2-weighted images show cardiac hydatid cyst with hyperintense centre surrounded by hypointense periphery (pericyst). Multiple intralesional daughter cysts are also seen.

\section{DISCUSSION}

Domestic dogs or other carnivores are the definitive hosts of echinococcal organisms. Sheep or other ruminant is the intermediate host ${ }^{7}$. Humans are infected accidentally when they eat unwashed and uncooked vegetables and swallow the ova of the parasite. Man acts as an intermediary carrier because the digested contaminated food released the hexacanth embryo of the parasite into the intestinal tract which is carried to the liver by the portal circulation ${ }^{8}$. The embryo develops into a cyst surrounded by the periparasitic host tissue (pericyst). The hydatid cyst has three layers: outer pericyst, middle ectocyst, and inner germinal layer (the endocyst), where the scolices (larval stage of the parasite) and the laminated membrane are produced. The cyst is covered by a multipotential germinal layer, which gives rise to the production of protoscolices and brood capsules (daughter vesicles) ${ }^{8}$. The terminal segment of the hermaphrodite called as proglottid, contains hundreds of eggs, which are discharged into the animal's intestine and expelled in its faeces ${ }^{9}$. The life cycle is completed when an infected intermediate host dies and its viscera, containing the larval form, are consumed by a definitive host ${ }^{7,9,10}$. Cardiac involvement of hydatid cyst is usually rare due to contractions of the heart, which provide a natural resistance to the viable cyst. Although hydatidosis may involve any part of the heart, the most common locations are the left ventricular wall $(60 \%)$ due to dominance of left coronary artery followed by the right ventricle (10\%), pericardium (7\%), left atrium (6-8\%), right atrium (3$4 \%)$, and the least being interventricular septum (4\%) ${ }^{11}$. Large cyst, as in our case, may cause displacement of the coronary vessels, rhythm disturbances and mechanical interference with the ventricular function ${ }^{12-14}$. Cardiac echinococcosis may prove fatal in the absence of surgical treatment ${ }^{15}$. Prognosis of these patients can be improved by extirpation of the lesion under cardiopulmonary bypass followed by antihelminthic drugs (mebendazole and albendazole) pre-operatively as well as post-operatively ${ }^{16,17}$.

Imaging is critical for the initial diagnosis, progression, and follow-up of echinococcosis. Electrocardiogram and chest radiograph can be abnormal. They are usually not reliable diagnostic methods ${ }^{1,2}$. Chest radiographs usually show a normal cardiothoracic ratio but in larger cardiac cysts, the ratio is increased ${ }^{18}$, as in our patient. Radiographic mass adjacent to the left ventricle signals the possibility that a cyst is a cardiac hydatid cyst. Electrocardiographic changes simulating arrhythmia or conduction defects due to compression of the bundle of His can be caused by cardiac hydatid cyst ${ }^{19-21}$. Electrocardiographic findings vary according to the sites of the cysts. The electrocardiogram shows QRS notching when cyst is in the interventricular septum, ST segment and T-wave alterations resembling arrhythmia when cyst is in left ventricle (as in our patient, T- wave inversion due to localization of cyst in left ventricular apex), lowvoltage $R$ waves will be seen in thinner cardiac wall area of parasite localization. The most effective and simple method of diagnosing hydatid cyst is transthoracic echocardiography ${ }^{22}$. On echocardiography, cyst will appear as oval echo-negative lesion with smooth contours. The appearance of the cysts on ultrasound ${ }^{23}$ varies based on the stage of the life cycle. On ultrasound, they are classified as follows:

(A) Cystic lesion: a simple cyst in the affected organ. This appearance is not diagnostic for echinococcosis.

(B) Active cysts: multiple cysts or septae are present in the parent cyst. 
(C) Transitional stage: daughter cysts may be present in the parent cyst, with hydatid sand or debris within the cyst. (The case described here was in the transitional stage.)

(D) Inactive stage: the cysts are echogenic and may be partially or completely collapsed on themselves.

In our case, diagnosis was suggested by sonography and transthoracic echocardiography which was confirmed by MRI and serologic tests in subsequent steps. Thoracic NCCT scan was performed but CT scan with contrast was not done because patient was allergic to contrast.

On CT their appearance varies: they may show a "spoke wheel" pattern or a water lily sign (where membranes appear within the matrix as serpentine linear structures, a finding that is highly specific for hydatid disease). Cystic wall calcification is best evident in CT. MRI appearance of a hydatid cyst depicts a characteristic round to oval

\section{REFERENCES}

1. Ameli M, Mobarhan HA, Nouraii SS. Surgical treatment of hydatid cysts of the heart: report of six cases. J Thorac Cardiovasc Surg. 1989;98(5 Pt 2):892901.

2. Salih OK, Celik SK, Topcuoglu MS, Kisacikoglu B, Tokcan A. Surgical treatment of hydatid cysts of the heart: a report of 3 cases and a review of the literature. Can J Surg. 1998;41:321-7.

3. Miralles A, Bracamonte L, Pavie A, Bors V, Rabago G, Gandjbakhch I, Cabrol C. Cardiac echinococcosis. Surgical treatment and results. J Thorac Cardiovasc Surg. 1994;107:184-90.

4. Abid A, Ben Omrane S, Kaouel K, Marghli A, Dhiab $\mathrm{M}$, Abid $\mathrm{N}$, et al. Intracavitary cardiac hydatid cyst. Cardiovasc Surg. 2003;11:521-5.

5. Maffeis GR, Petrucci O, Carandina R, Leme CA $J r$, Truffa M, Vieira $R$, et al. Cardiac echinococcosis. Circulation. 2000;101(11):1352-4.

6. Murphy $\mathrm{TE}$, Kean $\mathrm{BH}$, Venturin A,Lillihei CW. Echinococcus cyst of the left ventricle: report of a case with review of the pertinent literature. J Thorac Cardiovasc Surg. 1971;61:443-50.

7. Radford AJ. Hydatid disease. In: Weatherall DJ, Ledingharn JGG, Warrell DA, editors. Oxford Textbook of Medicine. Oxford: Oxford University Press; 1982.

8. Gormus N, Yeniterzi M, Telli HH, Solak H. The clinical and surgical features of right-sided intracardiac lesion appearing hypo-intense on T1-weighted images and hyper intense centre with hypo intense periphery (pericyst) on T2-weighted images.

\section{CONCLUSION}

The clinical presentation of cardiac hydatid cyst is usually insidious due to varying signs and symptoms and should be considered, particularly in endemic regions, in the differential diagnosis of patients with chest pain, even for those who do not have a history of hydatid disease. Furthermore, it should be noted that negative serology is found in up to $50 \%$ of cardiac locations. Echocardiography and MRI are easy, reliable, safe and effective imaging methods for establishing the diagnosis. The size, location and manifestations are the most important determinants of the disease management. Combination of surgical approach with albendazole therapy is the gold standard for Echinococcosis treatment. masses due to echinococcosis. Heart Vessels. 2004;19:121-4.

9. Manson-Bahr PEC, Apted FIC. Manson's tropical diseases. London: Bailiere Tindall; 1982. p 245-7.

10. Saidi F. Surgery of hydatid disease. Philadelphia: Saunders; 1976.

11. Dursun $M$, Terzibasioglu E, Yilmaz R, Cekrezi B, Olgar S, Nisli K, et al. Cardiac hydatid disease: CT and MRI findings. AJR Am J Roentgenol. 2008;190(1):226-32.

12. Rezaian GR, Aslani A. Endocardial hydatid cyst: a rare presentation of echinococcal infection. Eur J Echocardiogr. 2008;9:342-3.

13. Erol C, Candan I, Akalin H, Sonel A, Kervancio lu C. Cardiac hydatid cyst simulating tricuspid stenosis. Am J Cardiol. 1985;56:833-4.

14. Abbasi MD, Poorzand H, Zirak N, MD, Hosinikhah H. Chest pain due to compression effect of hydatid cyst on LAD: rare presentation of hydatid cyst in heart. Iranian Heart J. 2011;12:47-50.

15. Peters JH, Dexter L, Weiss S. Clinical and theoretical considerations of involvement of the left side of the heart with echinococcal cysts. Am Heart J. 1945;29:143.

16. Bryceson ADM, Cowie AGA, Macleod C, White $S$, Edwards D, Smyth JD, et al. Experience with mebendazole in the treatment of inoperable hydatid disease in England. Trans R Soc Trop Med Hyg. 1982;76(4):510-8. 
17. Beard TC, Rickard MD, Goodman HT. Medical treatment for hydatids. Med J Aust 1978; 1:633-635.

18. Muthu SK, Thiagarajan A, Govindarajan S, Coutinho CM, Gunawardena WJ, Swaminathan TS. Hydatid cyst of the cardiac interventricular septum: report of two cases. Br J Radiol. 2007;80(959):278-82.

19. Vestri A, Tardio R. Aspetti electrocardiografici dell'echinococcosi del cuore. Comunicazione tenuta alla Societa romana di chirurgia. 1968. EMES.

20. Heimann HL. Hydatid cyst in the heart. Br Med J. $1928 ; 1 ; 801$.
21. Ghanem MH, Darwish AE. Hydatid heart disease with paroxismal tachycardia. Br Heart J. 1951;13:109.

22. Oliver JM, Sotillo JF, Domínguez FJ, López de Sá E, Calvo L, Salvador A, et al. Two-dimensional echocardiographic features of echinococcosis of the heart and great blood vessels. Clinical and surgical implications. Circulation. 1988;78(2):327-37.

23. Shah DS, Parikh H, Shah B, Banuprakash S, Shah J. Imaging appearances of hydatid cyst. Ind J Radiol Imag. 2006;16(4):533-5. 